\title{
Icterícia grave e miocardiopatia tireotóxica com trombo atrial
}

\author{
Serious jaundice and thyrotoxic myocardiopathy with atrial thrombus
}

Marcus V. Leitão de Souza', Fernanda Satake Novaes'

${ }^{1}$ Serviço de Endocrinologia, Instituto Estadual de Diabetes e Endocrinologia Luiz Capriglione (IEDE), Rio de Janeiro, RJ, Brasil

Correspondência para:

Fernanda Satake Novaes Rua Antônio Cezarino, 572, ap. 112 13015-291 - Campinas, SP, Brasil fsatakenovaes@yahoo.com.br

Recebido em 18/Mar/2012 Aceito em 27/Jun/2012

\begin{abstract}
SUMÁRIO
Icterícia relacionada à tireotoxicose, e não como efeito das drogas antitireoidianas, é uma complicação rara que, em geral, ocorre na presença de insuficiência cardíaca (IC) ou hepatite. Apresentamos o caso de mulher de 54 anos de idade, branca, diagnóstico de hipertireoidismo por doença de Graves com icterícia associada a despeito da suspensão prévia de metimazol. A bilirrubina oscilava em valores elevados entre 30,0 e $52,3 \mathrm{mg} / \mathrm{dL}$, as transaminases eram pouco elevadas com valores de ALT, na admissão, de $46 \mathrm{U} / \mathrm{l}$ e AST de $87 \mathrm{U} / \mathrm{l}$; coagulograma e proteínas encontravam-se no limite inferior da normalidade, sendo TAP $68 \%$ e albumina $=2,5$ $\mathrm{g} / \mathrm{dL}$. Sorologias para hepatites foram negativas. Após a primeira dose de radioiodoterapia (RT), a bilirrubina atingiu seu valor máximo, que coincidiu com período de pior exacerbação da IC. A bilirrubina normalizou-se após quatro semanas da segunda dose de RT com a estabilização da IC e a normalização dos hormônios tireoidianos. Discutimos as possíveis etiologias de icterícia severa em pacientes hipertireoideos, assim como a difícil terapia anticoagulante com varfarina. Arq Bras Endocrinol Metab. 2012;56(7):456-60
\end{abstract}

\section{SUMMARY}

Jaundice related to thyrotoxicosis and not as an effect of antithyroid drugs is a rare complication that usually occurs in the presence of heart failure (HF) or hepatitis. We report a case of a 54-year-old white woman with hyperthyroidism caused by Graves's disease and jaundice despite methimazole suspension. Bilirubin fluctuated at high values, between 30.0 and $52.3 \mathrm{mg} / \mathrm{dL}$, transaminases were slightly increased, on admission $A L T=46 \mathrm{U} / \mathrm{L}$ and $\mathrm{AST}=87 \mathrm{U} / \mathrm{L}$; coagulation indices and serum proteins were on the lower limit of the normal range with PT $68 \%$ and albu$\min =2.5 \mathrm{~g} / \mathrm{dL}$. Serology for hepatitis was negative. After the first radioiodine therapy (RT), bilirubin reached its maximum, which coincided with the worst period of $\mathrm{HF}$ exacerbation. Bilirubin normalized 4 weeks after the second RT, with the stabilization of HF and normalization of thyroid hormones. We discuss the possible etiologies of severe jaundice in hyperthyroid patients, as well as the difficult anticoagulant therapy with warfarin. Arq Bras Endocrinol Metab. 2012;56(7):456-60

\section{INTRODUÇÃO}

A tireotoxicose pode apresentar repercussões clínicas em praticamente todos os sistemas orgânicos. Alterações hepáticas mais frequentes nestes pacientes estão relacionadas a efeitos hepatotóxicos das drogas antitiroidianas (DAT), no entanto, algumas alterações podem ser consequência direta da tireotoxicose. A disfunção hepática pode ocorrer em casos de tireotoxicose grave com desenvolvimento de hepatomegalia e icterícia, sendo descrita em alguns casos de doença prolongada, evoluindo inclusive com insuficiência hepática e óbito (1).
Avaliação diagnóstica das hepatites virais deve ser incluída já que estas podem cursar por longo período sem diagnóstico e predispor ao surgimento de hiperbilirrubinemia quando associada à tireotoxicose (2).

Apresentamos um caso de hipertireoidismo por doença de Graves com desenvolvimento de hiperbilirrubinemia grave e cardiopatia tireotóxica com fibrilação atrial e trombo no átrio esquerdo. Serão discutidas as possíveis causas dessa hiperbilirrubinemia, além das dificuldades para o manuseio da terapia anticoagulante com varfarina em um paciente com tireotoxicose $\mathrm{e}$ hepatopatia associada, ambos interferindo com a meiavida dos fatores vitamina $\mathrm{K}$ dependentes. 


\section{RELATO DE CASO}

Mulher branca, 54 anos, encaminhada ao Serviço de Endocrinologia do Instituto Estadual de Diabetes e Endocrinologia Luiz Capriglione (IEDE) devido a sinais e sintomas típicos de hipertireoidismo. A paciente apresentava histórico patológico pregresso de hipertensão arterial sistêmica, insuficiência cardíaca e fibrilação atrial, quando foi Instituído tratamento com metimazol na dose de $40 \mathrm{mg} /$ dia. No curso do tratamento, foram verificados níveis de bilirrubina elevados com quadro clínico de icterícia, colúria, fadiga e desconforto abdominal, o que levou à suspensão imediata da DAT e internação em outro nosocômio. Em função de hipertireoidismo descompensado, fibrilação atrial de alta resposta e derrame pleural bilateral, foi transferida para nosso serviço. $\mathrm{Na}$ admissão, a paciente apresentava-se orientada, hipocorada $2+/ 4+$, ictérica $3+/ 4+$, taquipneica $(26 \mathrm{irpm})$, normotensa $(120 \times 80 \mathrm{mmHg})$, eucárdica $(80 \mathrm{bpm})$, afebril e eutrófica (IMC $22,0 \mathrm{~kg} / \mathrm{m}^{2}$ ), estando na ocasião da admissão há três semanas sem uso de nenhuma DAT. A tireoide estava aumentada com aproximadamente $60 \mathrm{~g}$, consistência fibroelástica, sem nódulos ou sopros. A ausculta pulmonar evidenciou estertores bilaterais até um terço superior e a ausculta cardíaca, ritmo cardíaco irregular sem sopros. Apresentava abdome sem massas ou visceromegalias palpáveis. Em membros inferiores, notava-se edema com cacifo $2+/ 4+$ sem sinais de flogose e panturrilhas livres. Os exames laboratoriais na internação encontram-se na tabela 1 .

Tabela 1. Exames laboratoriais da admissão hospitalar

\begin{tabular}{lcc}
\hline & Resultado & Valor de referência \\
\hline T4 livre & 8,76 & 0,8 a $1,8 \mathrm{ng} / \mathrm{dl}$ \\
TSH & TSH $<0,01$ & $0,35 \mathrm{a} 5,5 \mu \mathrm{U} / \mathrm{mL}$ \\
Albumina & 2,5 & $3,5-5,5 \mathrm{~g} / \mathrm{dl}$ \\
TAP & $68 \%$ & Acima de $70 \%$ \\
ALT & 46 & Até $31 \mathrm{U} / \mathrm{l}$ \\
AST & 87 & Até $31 \mathrm{U} / \mathrm{l}$ \\
Fosfatase alcalina & 226 & $35-104 \mathrm{U} / \mathrm{l}$ \\
GGT & 138 & $7-32 \mathrm{U} / \mathrm{l}$ \\
BB total & 44 & Até $1 \mathrm{mg} / \mathrm{dL}$ \\
BB indireta & 35,74 & Até $0,2 \mathrm{mg} / \mathrm{dL}$ \\
BB direta & 8,26 & Até $0,8 \mathrm{mg} / \mathrm{dL}$ \\
\hline
\end{tabular}

T4 livre: tiroxina livre; TSH: hormônio tireoestimulante; TAP: tempo de protrombina; ALT: alanina aminotransferase; AST: aspartato aminotransferase; GGT: gama glutamil transpeptidase; BB: bilirrubina.

A ultrassonografia abdominal mostrou fígado com volume nos limites da normalidade, contornos regula- res e sem lesões focais definidas ao método; ausência de dilatação de vias biliares intra ou extra-hepáticas; vesícula biliar com parede de espessura preservada.

O eletrocardiograma evidenciou fibrilação atrial com resposta ventricular média de 60 sístoles por minuto e bloqueio de ramo esquerdo de $3^{\circ}$ grau.

No ecocardiograma transesofágico, foi encontrado aumento de ambos os átrios e do diâmetro sistólico do ventrículo esquerdo que apresentava discreta hipocinesia difusa de suas paredes, função sistólica do ventrículo esquerdo levemente diminuída, fração de ejeção de $69 \%$, e pequeno trombo organizado em auriculeta esquerda (Tabela 2).

Tabela 2. Parâmetros ecocardiográficos

\begin{tabular}{lcc}
\hline & & Valores de referência \\
\hline VE sístole & $3,8 \mathrm{~cm}$ & Até $3,7 \mathrm{~cm}$ \\
VE diástole & $5,6 \mathrm{~cm}$ & Até $5,6 \mathrm{~cm}$ \\
VD & $2 \mathrm{~cm}$ & Até $3,0 \mathrm{~cm}$ \\
AE & $4,9 \mathrm{~cm}$ & Até $4,0 \mathrm{~cm}$ \\
FE & $69 \%$ & $>53 \%$ \\
\hline
\end{tabular}

Septo interatrial íntegro, ausência de remora atrial, baixa velocidade de fluxo $(<0,20 \mathrm{~m} / \mathrm{s}) \mathrm{com}$ pequeno trombo organizado em auriculeta esquerda.

Moderada regurgitação mitral ao ecoDoppler.

Leve regurgitação aórtica e tricúspide.

Função sistólica de VE discretamente reduzida.

VE sístole: diâmetro sístole do ventrículo esquerdo; VE diástole: diâmetro diastólico do ventrículo esquerdo; VD: tamanho do ventrículo direito; AE: tamanho do átrio esquerdo; FE: fração de ejeção; auriculeta esquerda = apêndice atrial esquerdo.

Foram realizadas sorologias para hepatites virais A, $\mathrm{B}$ e $\mathrm{C}$, sendo todas não reagentes.

Terapia com carvedilol, furosemida e espironolactona foi instituída para compensação do quadro de insuficiência cardíaca e anticoagulação com varfarina sódica. A terapia com lítio na dose de $600 \mathrm{mg} /$ dia foi iniciada uma semana antes da radioiodoterapia e mantida até seis dias depois a fim de amenizar a descompensação do hipertireoidismo. Foi instituído também tratamento com colestiramina conforme sugerido por Solomon e cols. (3) visando ao declínio mais rápido e efetivo dos hormônios tireoidianos. Com a compensação do quadro de insuficiência cardíaca, foi realizada dose terapêutica com iodo radioativo com atividade de $17 \mathrm{mCi}$ de $\mathrm{I}^{131}$ com recuperação temporária (Tabela 3 ). Cinco meses depois, a recrudescência do quadro de hipertireoidismo justificou um novo tratamento com $25 \mathrm{mCi}$ de $\mathrm{I}^{131}$. Os maiores níveis de bilirrubina foram encontrados nos momentos de exacerbação da insuficiência cardíaca, alcançando o máximo de $52,3 \mathrm{mg} / \mathrm{dL}$, estando 
Tabela 3. Função hepática e função tireoidiana antes e após sessões de radioiodoterapia

\begin{tabular}{|c|c|c|c|c|c|c|c|}
\hline & & \multicolumn{2}{|c|}{$17 \mathrm{mCi}$} & \multirow{2}{*}{$\begin{array}{c}\begin{array}{c}\text { Remissão } \\
\text { temporária }\end{array} \\
13 / 11 / 2007\end{array}$} & \multirow{2}{*}{$\begin{array}{c}25 \mathrm{mCi} \\
28 / 1 / 2008\end{array}$} & \multirow{2}{*}{$\begin{array}{c}\begin{array}{c}\text { Avaliação } \\
\text { pós-RI }\end{array} \\
1 / 4 / 2008\end{array}$} & \multirow{2}{*}{ 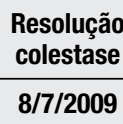 } \\
\hline & $17 / 9 / 2007$ & $24 / 9 / 2007$ & $16 / 10 / 2007$ & & & & \\
\hline BB total & 44 & 40,70 & 12,6 & 2,8 & 52,3 & - & 1,03 \\
\hline BB indireta & 8,26 & 14,40 & 2,9 & 0,59 & 12,08 & - & 0,57 \\
\hline BB direta & 35,74 & 26,30 & 9,7 & 2,21 & 40,22 & - & 0,46 \\
\hline Albumina & 2,5 & 5,0 & 2,2 & 3,5 & 2,9 & - & 4,5 \\
\hline TAP & $68 \%$ & $69 \%$ & $60,9 \%$ & $87 \%$ & $43,8 \%$ & $33,4 \%$ & \\
\hline INR & 1,3 & 1,27 & 1,42 & 1,10 & 1,88 & - & \\
\hline TSH & $<0,01$ & - & 0,01 & 6,11 & 0,02 & 0,01 & \\
\hline T4 livre & 8,07 & - & 5,10 & $<0,011$ & 8,58 & 7,06 & \\
\hline AST & 87 & 97 & 35 & 29 & 85 & & 25 \\
\hline ALT & 46 & 52 & 15 & 19 & 62 & & 11 \\
\hline GGT & & & 89 & 219 & 76 & & 44 \\
\hline
\end{tabular}

Valores de referência: BB (bilirrubina) total: até $1 \mathrm{mg} / \mathrm{dL} ; \mathrm{BB}$ indireta até $0,2 \mathrm{mg} / \mathrm{dL} ; \mathrm{BB}$ direta até $0,8 \mathrm{mg} / \mathrm{dL} ;$ albumina 3,5-5,5 g/dL; TAP (tempo de protrombina): > de 70\%; INR: 0,9 a 1,1; TSH 0,35 a $5,5 \mu \mathrm{U} / \mathrm{mL} ; \mathrm{T} 4$ livre 0,8-1,8 $\mathrm{ng} / \mathrm{dL} ;$ ALT até $41 \mathrm{U} / \mathrm{L}$ para homens e até 31 para mulheres; AST até 37 para homens e até 31 para mulheres.

a paciente em tireotoxicose com TSH de $0,02 \mu \mathrm{U} / \mathrm{mL}$ e T4 livre de $8,58 \mathrm{ng} / \mathrm{dL}$. A resolução da icterícia ocorreu 28 semanas após suspensão do metimazol e quatro semanas após a última dose de $25 \mathrm{mCi}$ de $\mathrm{I}^{131}$ (Tabela 3).

A varfarina foi iniciada conjuntamente à heparina conforme o protocolo de anticoagulação (4) e, posteriormente, mantida sem heparina associada em doses entre $1,25 \mathrm{mg} /$ dia e $7,5 \mathrm{mg} /$ dia. Não houve correlação direta entre o status tireoidiano e a dose do anticoagulante, tendo a paciente alcançado função tireodiana próxima do normal em determinados momentos, mesmo apresentando tempo de atividade de protrombina (TAP) incoagulável com INR > 9 em vigência de 5 $\mathrm{mg} /$ dia de varfarina.

As sorologias para hepatite autoimune foram negativas.

Paciente evoluiu com hipotireoidismo e compensação da insuficiência cardíaca, porém recusou-se a tentar cardioversão elétrica da fibrilação atrial. Após dois anos e seis meses de hipotireoidismo compensado, apresentou reversão espontânea para ritmo sinusal. Foi suspensa terapia anticoagulante com varfarina após quatro semanas de ritmo sinusal confirmado por eletrocardiograma.

\section{DISCUSSÃO}

Hiperbilirrubinemia grave em paciente com hipertireoidismo é uma condição rara e que geralmente ocorre na presença de insuficiência cardíaca ou doença hepática em atividade. $\mathrm{O}$ caso em questão é peculiar em função dos níveis atingidos de bilirrubinas bem acima do que foi descrito em casos publicados na literatura e provavelmente ocorreu devido à associação da tireotoxicose à cardiopatia com congestão hepática e manutenção da hiperbilirrubinemia por mais de seis meses após a suspensão do metimazol. Além disso, a presença concomitante de insuficiência cardíaca e fibrilação atrial tornou a anticoagulação muito mais desafiadora em função da hepatopatia e das oscilações hormonais tireoidianas.

Gurioli e cols. relataram um caso de hipertireoidismo e icterícia em um homem de 63 anos em que os níveis de bilirrubina alcançaram níveis de $27,41 \mathrm{mg} / \mathrm{dL}$. Hepatites virais e autoimunes foram excluídas, assim como insuficiência cardíaca. O paciente apresentou melhora do quadro com uso de metimazol e os autores destacam que os níveis de bilirrubina alcançados foram bastante elevados considerando ausência de insuficiência cardíaca, na qual, em geral, ocorrem elevações maiores da bilirrubina diante de um quadro de hipertireoidismo (5).

Hull e cols. descreveram dois casos de hipertireoidismo grave associado à icterícia em mulher de 38 anos e outro em uma mulher de 35 anos em que os níveis de bilirrubina foram de 35,2 e $42 \mathrm{mg} / \mathrm{dL}$, respectivamente, na ausência de doença hepática, exposição à DAT ou insuficiência cardíaca congestiva. Ambas apresentaram resolução da icterícia com uso de DAT, o que levou os autores a concluir que não se deve tardar o uso dessas medicações uma vez que outras doenças hepáticas sejam excluídas (6). 
O hormônio tireoidiano é importante para a função hepática normal e o metabolismo da bilirrubina e, em contrapartida, o fígado possui importante papel no metabolismo desse hormônio. Estudos de necropsia têm mostrado inflamação hepática, necrose centrolobular e fibrose em pacientes com hipertireoidismo. A fisiopatologia desses achados não está totalmente esclarecida. Acredita-se que o excesso de hormônio tireoidiano leva a um maior dano oxidativo dos tecidos consequente ao aumento da taxa metabólica (5), ou seja, a diminuição da taxa de oxigênio e o aumento do fluxo hepático de sangue parecem ser os responsáveis pela colestase por interferência no transporte de bile (7). $\mathrm{O}$ efeito tóxico direto da tiroxina (T4) sobre os hepatócitos e a alteração na fluidez das membranas canaliculares causadas pelo hipertireoidismo poderiam também explicar a colestase por ação na atividade transmembrana da enzima Na-K-ATPase (2).

Hepatotoxicidade relacionada à DAT é sempre a primeira hipótese principalmente por sua maior frequência quando comparada a um quadro de hepatite colestática pela tireotoxicose. Colestase secundária ao uso de metimazol surge em torno de 30 dias após início do uso e persiste por, no máximo, 8 a 10 semanas após interrupção da medicação (6). No caso em questão, a colestase teve melhora (sem completa resolução) após a primeira dose de iodo radioativo e novo pico de elevação após a segunda dose com resolução completa somente após 28 semanas da última administração do metimazol. Os picos de hiperbilirrubinemia coincidem com agravamento da tireotoxicose e da insuficiência cardíaca, tornando, assim, remota a possível associação da colestase com o uso prévio de metimazol.

Em relação à hepatite autoimune, o quadro clínico é bastante variável com início da doença, podendo ser insidioso ou abrupto, mas, na paciente em questão, a não elevação significativa de transaminases (normalmente mais que 10 vezes o limite superior), o aumento de fosfatase alcalina, o histórico de uso de medicação potencialmente hepatotóxica e anticorpos negativos afastam a possibilidade desse diagnóstico (7).

Outra possibilidade como causa da colestase seria a insuficiência cardíaca (IC). São ainda pouco conhecidas as alterações de pequena intensidade nos testes de função hepática resultantes de graus menos graves de insuficiência cardíaca e também sobre suas manifestações clínicas (8). As anormalidades enzimáticas melhoram com a compensação da insuficiência cardíaca e a icterícia leve ocorre em um terço dos pacientes (9). Um estudo que buscou relacionar débito cardíaco e anormalidades da função hepática mostrou que aumentos significativos de transaminases hepáticas foram observados nos pacientes com muito baixo débito (menor ou igual a $\left.1,5 \mathrm{~L} / \mathrm{min} / \mathrm{m}^{2}\right)$. A análise da fosfatase alcalina e gamaglutamiltransferase ( $\gamma$-GT) mostrou um aumento progressivo dos níveis séricos dessas enzimas com a piora da IC, corroborando que esse perfil colestático de alterações laboratoriais se correlaciona com a gravidade da IC $(10,11)$. Outro mecanismo para explicar a disfunção hepática decorrente da IC seria o aumento da pressão mecânica dentro dos sinusoides hepáticos explicando a lesão das células endoteliais. Estudos de microscopia já comprovaram essas alterações em ratos (12) submetidos à obstrução do retorno venoso hepático e alterações semelhantes têm sido relatadas em seres humanos com colestase extra-hepática.

Outra difícil questão se refere à anticoagulação diante de um quadro de fibrilação atrial. $\mathrm{O}$ manuseio da varfarina não é simples, tornando-se ainda mais complexo diante do hipertireoidismo que teoricamente acelera o metabolismo dos fatores vitamina $\mathrm{K}$ dependentes com necessidade de doses inferiores de varfarina (13). No entanto, nossa paciente, mesmo com estabilização do hipertireoidismo e com a mesma dose de varfarina, apresentou INR > 9 com sério risco de complicações hemorrágicas. A hepatopatia associada torna o uso de varfarina mais amedrontador, no entanto, em função da presença de trombo atrial visualizado ao ecocardiograma transesofágico, não houve alternativa em função do alto risco de complicação embólica. Em casos de hipertireoidismo com fibrilação atrial, realizamos ecocardiograma transesofágico conforme sugerido por Souza e cols. (14) a fim de auxiliar na decisão de tromboprofilaxia e, possivelmente, evitar o uso de varfarina nos casos em que não são visualizados trombos no átrio, o que efetivamente não foi possível no caso em questão.

Concluímos que a disfunção hepática nem sempre decorre do uso de DAT, sendo inicialmente importante o diagnóstico diferencial com as hepatites virais e autoimune. No entanto, a associação de hipertireoidismo com insuficiência cardíaca congestiva deve ser lembrada como uma possível causa de icterícia nos pacientes com mais de 8-10 semanas de suspensão das DAT e sem evidências de hepatite por vírus ou autoimune.

Declaração: os autores declaram não haver conflitos de interesse científico neste estudo. 


\section{REFERÊNCIAS}

1. Kronenberg HM, Melmed S, Polonsky KS, Larsen PR. Williams Textbook of Endocrinology. 11.ed. Philadelphia: Saunders Elsevier; 2008.

2. Chawla M, Bal CS. Four cases of coexistent thyrotoxicosis and jaundice: results of radioiodine treatment and a brief review. Thyroid. 2008;18(3):289-92.

3. Solomon BL, Wartofsky L, Burman KD. Adjunctive cholestyramine therapy for thyrotoxicosis. Clin Endocrinol (Oxf). 1993;38(1):39-43.

4. Zimerman LI, Fenelon G, Martinelli Filho M, Grupi C, Atié J, Lorga Filho A, et al. Sociedade Brasileira de Cardiologia. Diretrizes Brasileiras de Fibrilação Atrial. Arq Bras Cardiol. 2009;92(6 supl.1):1-39.

5. Gurioli L, Babini G, Rizzi R, Bertello P. Hyperthyroidism and cholestasis: a case report. Ann Ital Med Int. 1998;13(4):244-7.

6. Hull K, Horenstein R, Naglieri R, Munir K, Ghany M, Celi FS. Two cases of thyroid storm-associated cholestatic jaundice. Endocr Pract. 2007;13(5):476-80.

7. Dienstag JL. Chronic hepatitis. In: Fauci AS, Braunwald E, Kasper DL, Hauser SL, Longo DL, Jameson JL, et al. Harrison's Principles of Internal Medicine 17.ed. NewYork: McGraw-Hill; 2008. p. 1955-68.
8. Vasconcelos LABA, Almeida EA, Bachur LF. Avaliação clínica e laboratorial hepática em indivíduos com insuficiência cardíaca congestiva. Arq Bras Cardiol. 2007;88 (5):590-5.

9. Naschitz JE, Slobodin G, Lewis RJ, Zuckerman E, Yeshurun D. Heart diseases affecting the liver and liver diseases affecting the heart. Am Heart J. 2000;1:140.

10. Kubo $\mathrm{SH}$, Walter BA, John DH, Clark M, Cody RJ. Liver function abnormalities in chronic heart failure. Influence of systemic hemodynamics. Arch Intern Med. 1987;147(7):1227-30.

11. Lau GT, Tan HC, Kritharides L. Type of liver dysfunction in heart failure and its relation to the severity of tricuspid regurgitation. Am J Cardiol. 2002;90:1405-9.

12. Cogger VC, Fraser R, Le Couteur DG. Liver dysfunction and heart failure. Am J Cardiol. 2003;91:1399.

13. Ansell J, Hirsh J, Hylek E, Jacobson A, Crowther M, Palareti G; American College of Chest Physicians. Pharmacology and management of the vitamin K antagonists: American College of Chest Physicians Evidence-Based Clinical Practice Guidelines (8th Edition). Chest. 2008;133(6):160S-98S.

14. de Souza MV, Duarte MM, Coeli CM, Vaisman M. Atrial fibrillation \& hyperthyroidism: relation between transesophageal markers of a thrombogenic milieu and clinical risk factors for thromboembolism. Clin Endocrinol (Oxf). 2012;76(3):448-53. 